\title{
LINE-OF-SIGHT INTEGRATION: A POWERFUL TOOL FOR VISUALIZATION OF THREE-DIMENSIONAL SCALAR FIELDS
}

\author{
H. RUDER, T. ERTL, F. GEYER, H. HEROLD and U. KRAUS \\ Lehrstuhl für Theoretische Astrophysik der Universität Tübingen, Auf der Morgenstelle $12 \mathrm{C}$. \\ D.7400 Tubingen. Federal Republic of Germany
}

\begin{abstract}
An easy conceivable but very powerful method for the visualization of three-dimensional scalar fields is described. The way this method works is illustrated by some examples and the computed pictures are compared with the results of other methods of representation.
\end{abstract}

\section{INTRODUCTION}

The numerical modelling as well as the experimental monitoring of complex physical systems in many cases produce as a result the discrete values of a three-dimensional scalar field $\Phi(x, y, z)$, for instance a density, a temperature or a probability distribution in space. which has to be adequately visualized in order to allow a physical interpretation.

The traditional methods of presenting such results are lines of constant values in different orthogonal cutting planes which can additionally be colour-coded or three-dimensional plots of the corresponding contour surfaces. The method which is presented here can convey a genuine impression of the three-dimensional structure of a scalar field and seems to be widely ap-

Research supported in pan by the Deutsche Forschungs. gemeinschaft (DFG).

Send offprint requests to: H. Ruder plicable in the field of Visualization in Scientific Computing [1].

\section{DESCRIPTION OF THE METHOD}

The basic idea is that the three-dimensional scalar field $\Phi$ emits "light" with an intensity which is proportional to the local numerical values of the scalar field and which can penetrate it without absorption. The scalar field is therefore equivalent to an "optically thin self-radiating gaseous nebula." The intensity which a distant observer receives from such an object out of a specific direction is just the integral along the lineof-sight of the intensities emitted towards the observer. By scanning the whole object with the line-of-sight moving across it in a fine grid a raster image can be computed. which is a good approximation of what the observer actually sees. In Fig. I the procedure is schematically illustrated and the notation is explained. For

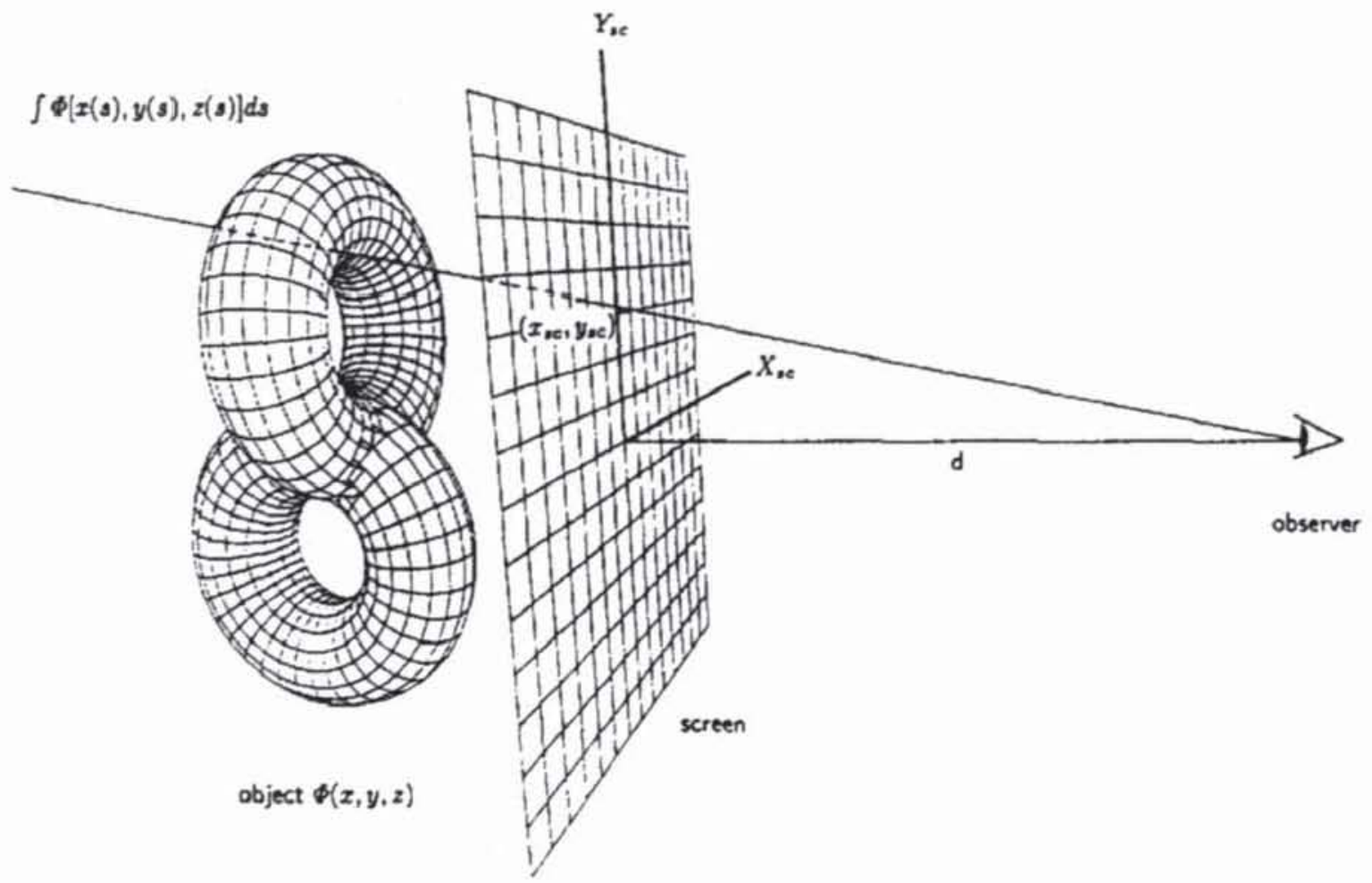

Fig 1. Schematic representation of the method of line-of-sight integration. For each pixel on the screen the intensity is set proportional to the integral through the object along the line-of-sight. 


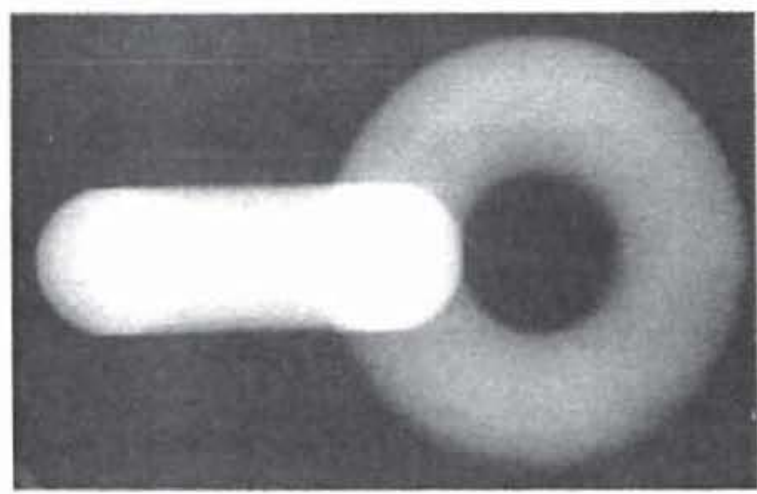

(a)

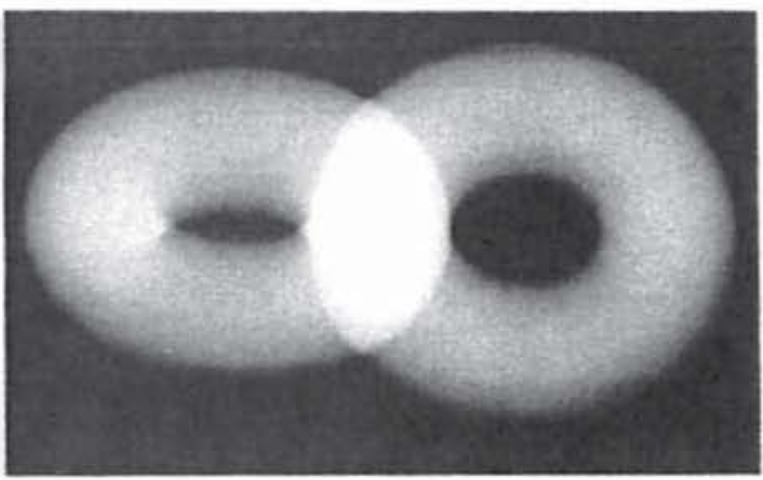

(b)

Fig 2. The result of line-of-sight integration for two intersecting tori with a homogeneous intensity distribution inside. The three-dimensional structure is discretized in a $200 \times 200 \times 200$ cube. The pictures on the screen have a resolution of $500 \times 300$ pixels and 256 intensity levels. The tori in (b) are rotated $35^{\circ}$ around the line which connects the centers of the tori.

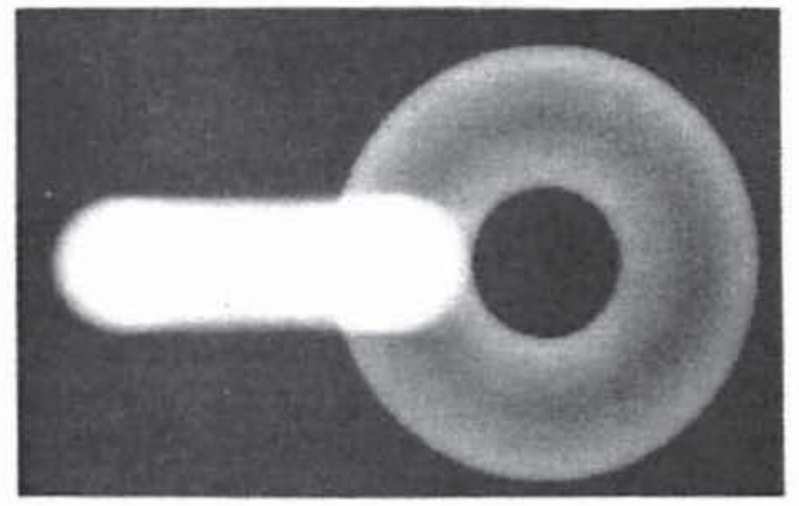

(a)

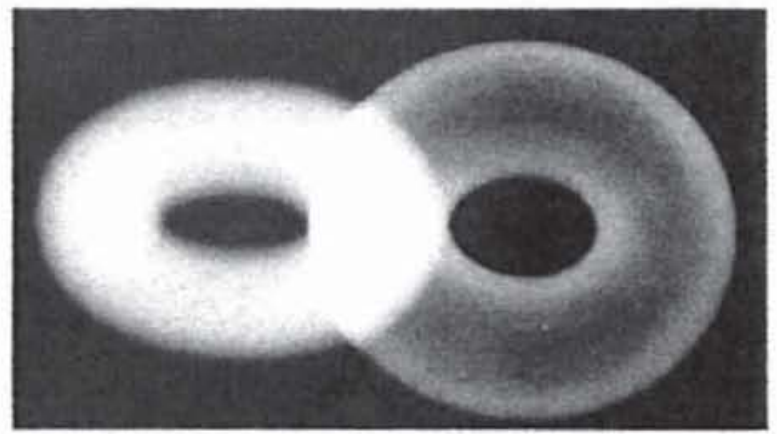

(b)

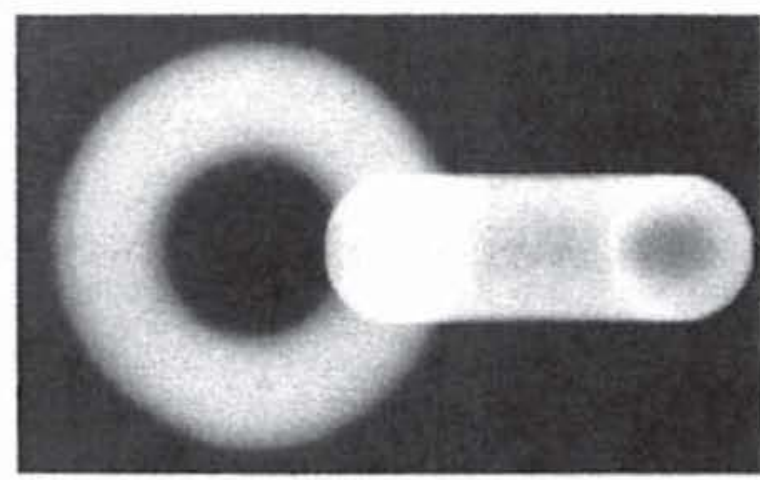

(c)

Fig. 3. The same situation as in Fig 2 but with an intensity distribution of the form $I(r)=I_{0}\left[1-\left(r / r_{r}\right)^{3}\right]$ (increasing towards the center) for the left torus and $I(r)=\left(r / r_{r}\right)^{3}$ (decreasing towards the center) for the right one ( $r_{r}$ is the radius of the generating circle and $\left.0 \leq r \leq r_{\tau}\right)$. The rotational angles with respect to the first picture (a) are $35^{\circ}(\mathrm{b})$ and $90^{\circ}(\mathrm{c})$. 

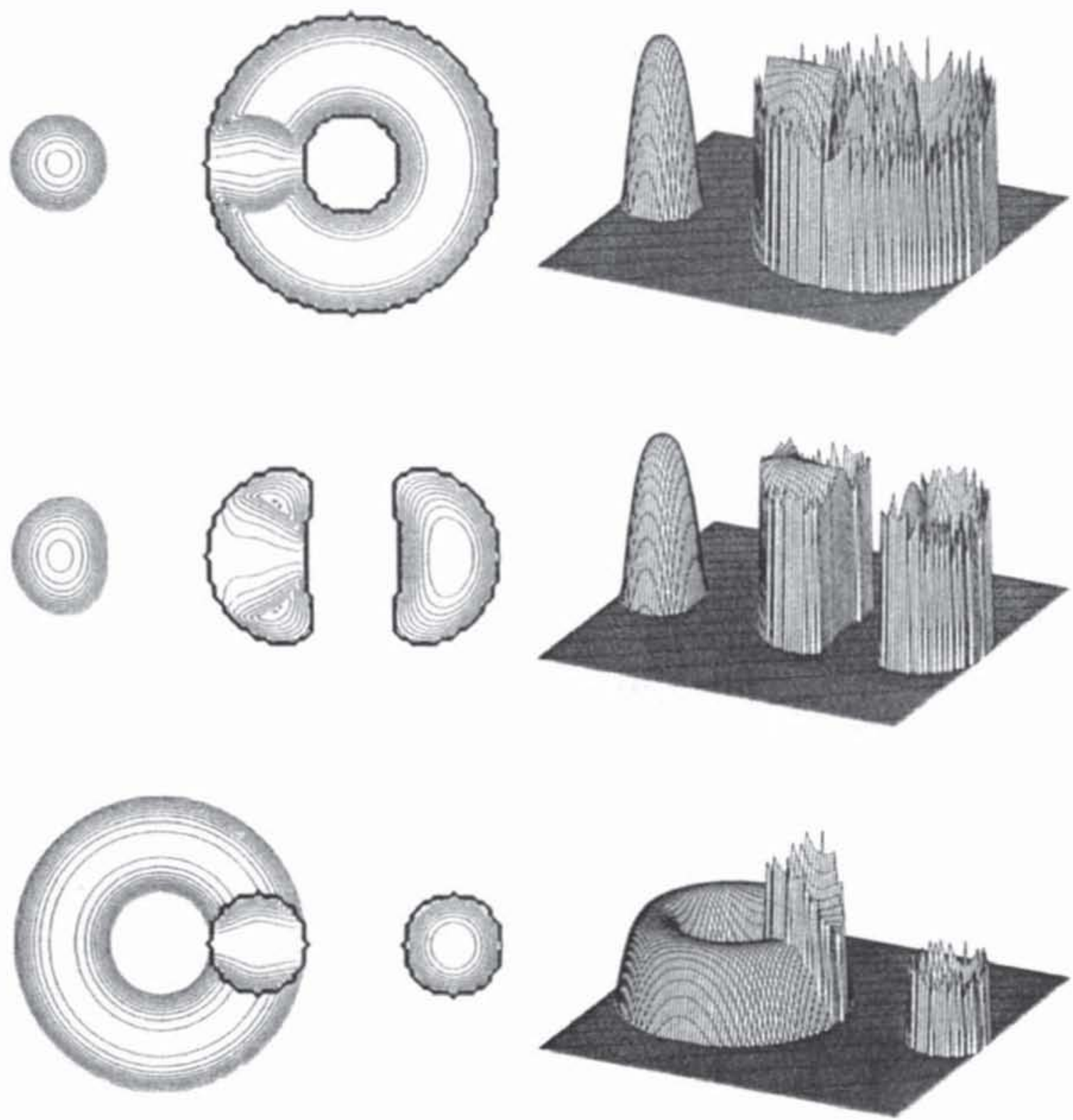

Fig 4. Isophotes in cuts which are parallel to the picture planes of Fig 3 and contain the centres of the two tori (left) and the corresponding contour surfaces (right). (The jagged ridges are caused by shortcomings of the plot routine used.)

a realistic impression a finite distance $d$ of the observer is appropriate (central projection). The intensity $I$ as a function of the coordinates $x_{k}, y_{x}$ on the screen is given by

$$
I\left(x_{*}, y_{*}\right)=\int_{s-\infty}^{s} \Phi[x(s), y(s), z(s)] d s,
$$

where $s$ is the are length of the line-of-sight. With the orientation of the axes defined in Fig. 1, the cartesian space coordinates $x, y, z$ depend on $s, x_{k}, y_{\kappa}$, and $d$ in the following form

$$
\begin{aligned}
& x(s)=x_{k \kappa}\left(1+s / s_{\kappa}\right) \\
& y(s)=y_{k x}\left(1+s / s_{\alpha}\right) \\
& z(s)=-d s / s_{\alpha}
\end{aligned}
$$

with the abbreviation $s_{x}=\sqrt{x_{w}^{2}+y_{w}^{2}+d^{2}}$.
The two-dimensional field $I\left(x_{k x}, y_{x}\right)$ can immediately be mapped onto the bit planes of a raster graphics display using either an intensity- or a colour-coding scheme and yields a true picture of the self-radiating object. We would like to demonstrate the visualization power by comparing it with different methods by means of some examples.

\section{EXAMPLES}

\section{a. Intersecting orthogonal tori}

We first consider two intersecting orthogonal tori, which are approximated by discretizing the corresponding three-dimensional structure in a $200 \times 200$ $\times 200$ cube. For all pictures of the tori we have chosen a resolution of $500 \times 300$ pixels and 256 intensity leveis. In Fig. 2 the intensity distribution inside the tori is homogeneous and they are shown at two different orientations. In Fig. 3 the intensity distribution is of 


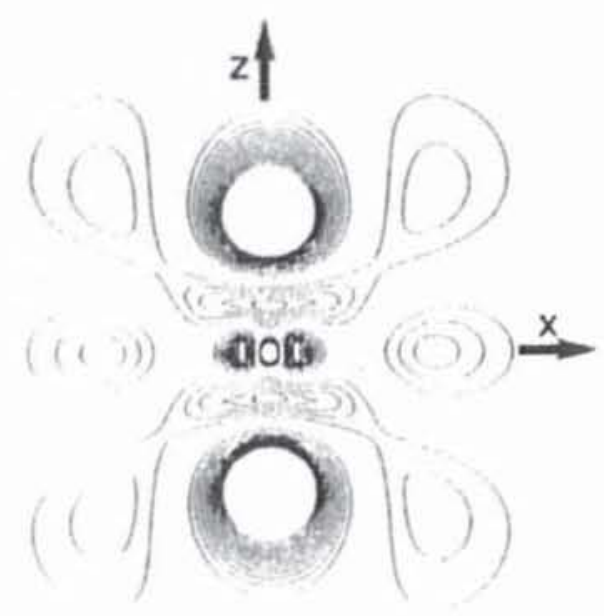

y

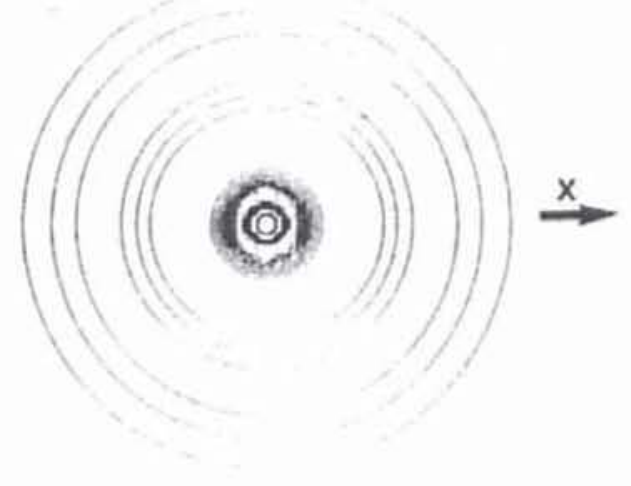

(a)

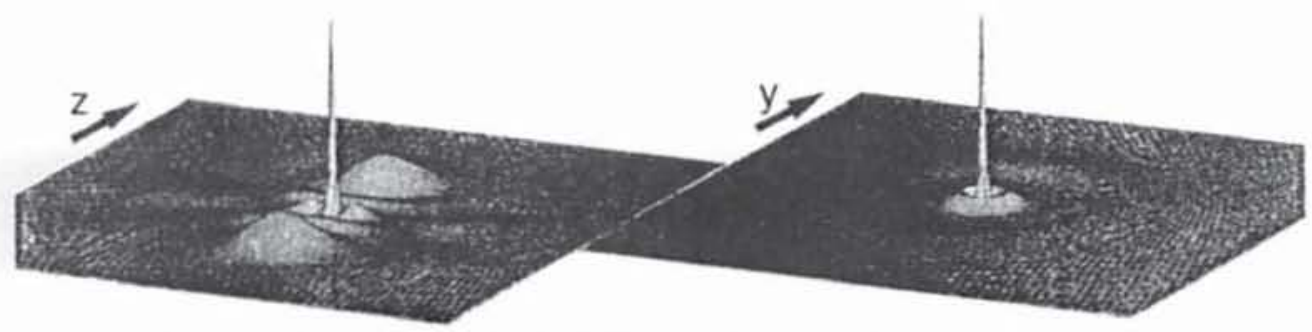

(b)
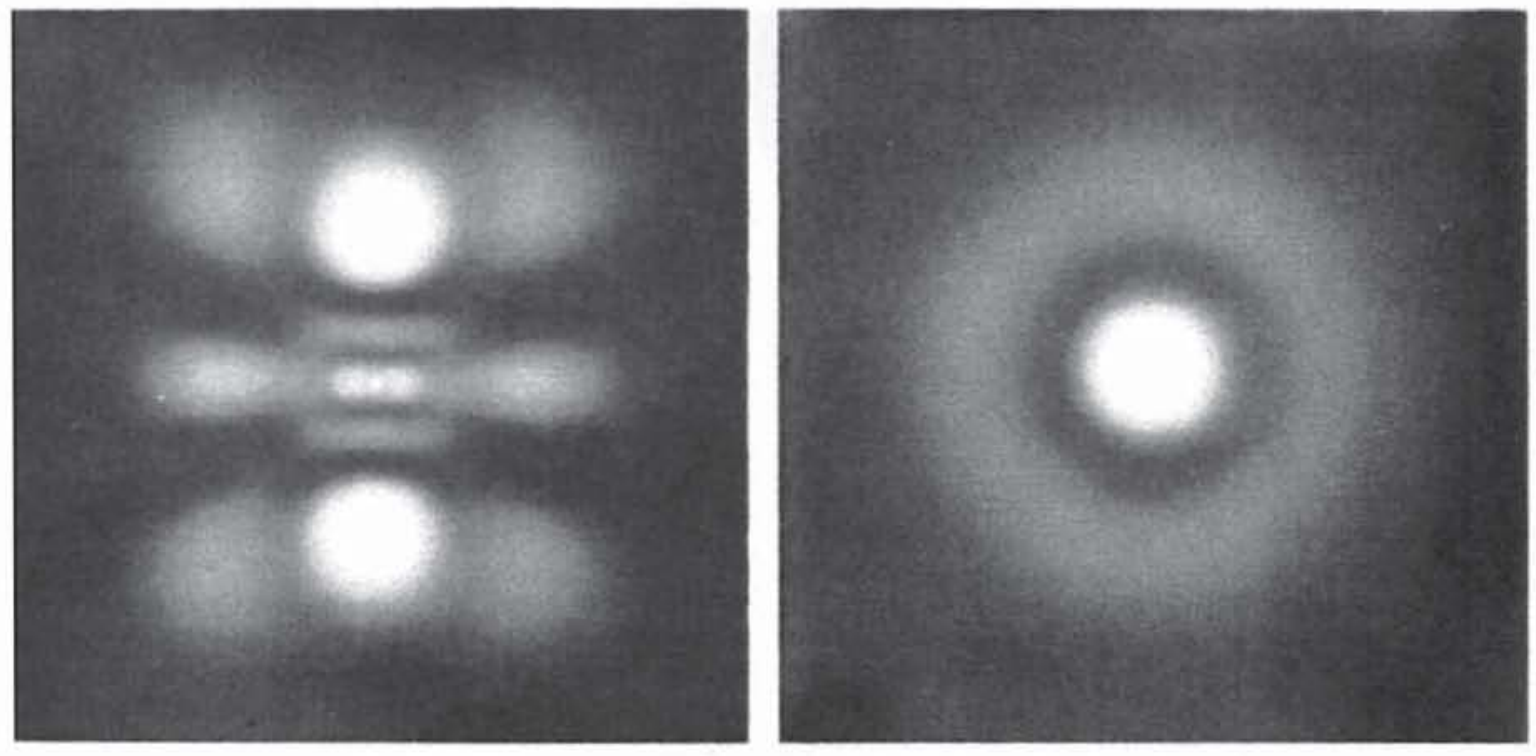

(c)
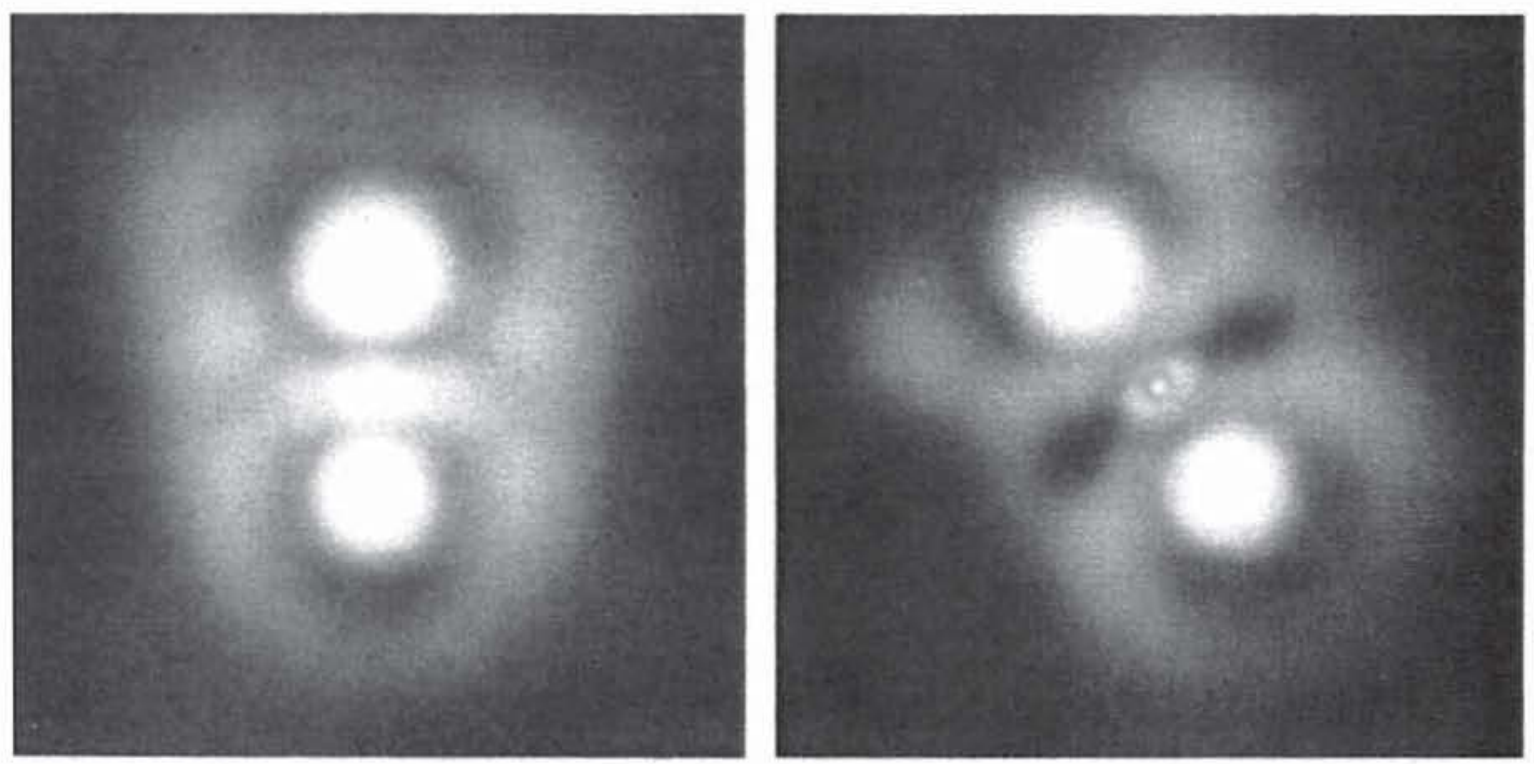

(d)

Fig. 5. Probability of presence of the electron in an excited state of the hydrogen atom (principal quantum number $n=5$, magnetic quantum number $m=0$ ). Lines of constant probability density (a), the corresponding contour surfaces (b) and the results of the line-of-sight integration (c, d). In (d) the atom is rotated by $45^{\circ}$ around the $x$-axis (left) and additionally by $45^{\circ}$ around the $z$-axis (right). The real extent of the atom in this state is about $3 \mathrm{~nm}$. 

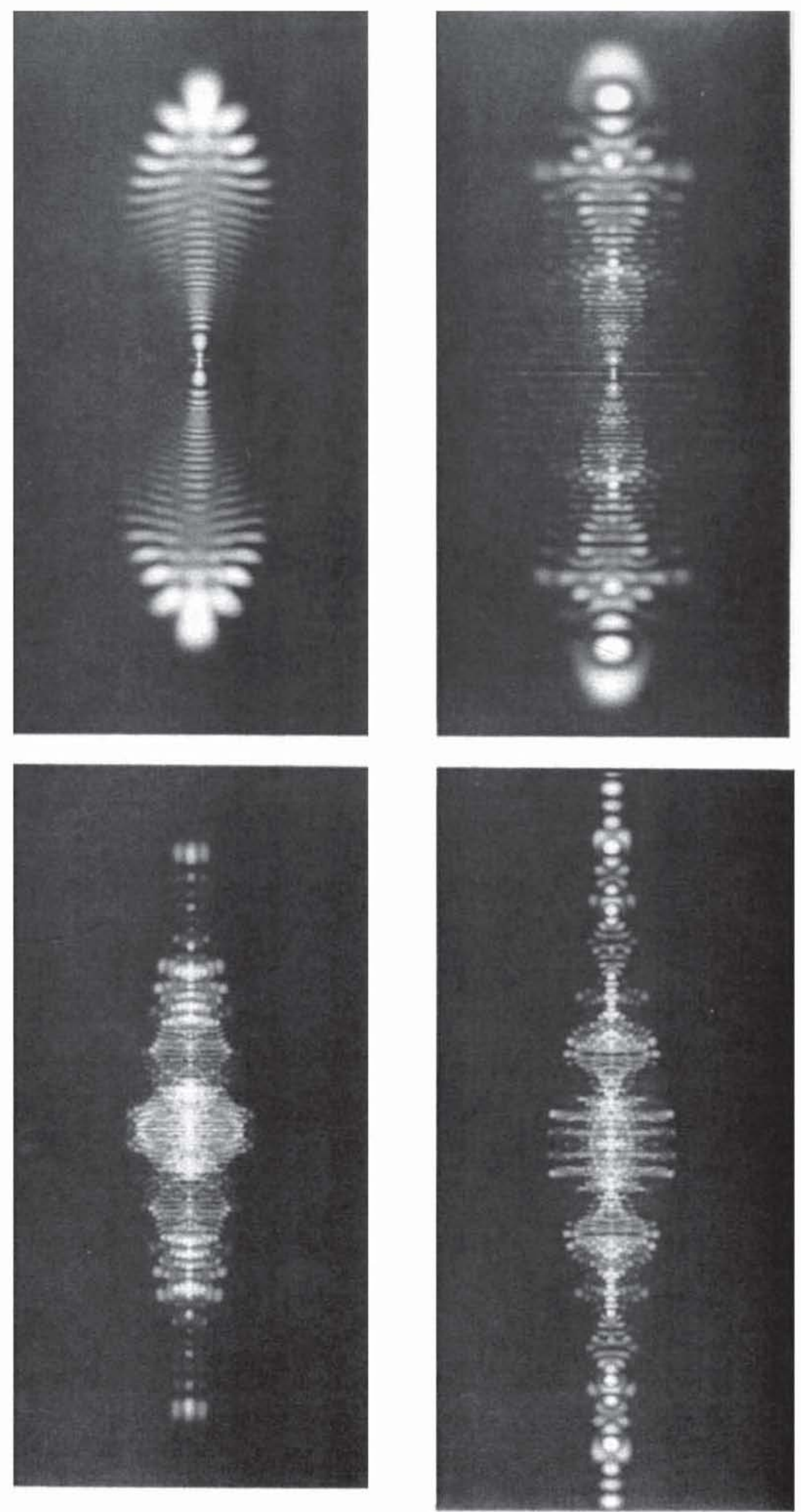

Fig 6. Probability of presence of the electron in highly excited (Rydberg) states of the hydrogen atom in a magnetic field of 6 Tesla. These bizarre atomic structures have a spatial extent of the order of $10^{-3} \mathrm{~mm}$. 
the form $I(r)=I_{0}\left[1-\left(r / r_{r}\right)^{3}\right]$ for the left torus and $I(r)=I_{0}\left(r / r_{T}\right)^{3}$ for the right torus, where $r_{T}$ is the radius of the generating circle and $0 \leq r \leq r_{r}$. The rotational angies with respect to the first picture (a) are $35^{\circ}$ (b) and $90^{\circ}$ (c). In the left half of Fig. 4 we have plotted the isophotes in cuts which are parallel to the picture planes of Fig. 3 and contain the centres of the two tori, in the right half of Fig. 4 the corresponding contour surfaces are shown in threedimensional plots. (The jagged ridges are caused by shortcomings of the plot routine used.) While the lineof-sight integration immediately gives intuitively understandable pictures, the other methods of representation cannot convey the correct impression of the global three-dimensional structure.

\section{b. The hydrogen atom}

Here the three-dimensional scalar field is the squared absolute value $|\Psi|^{2}$ of the wave function $\Psi(r)$ which is the probability of presence of an electron. Using spherical polar coordinates $r, \vartheta, \varphi$ and the Bohr radius $a_{0} \approx 0.529 \cdot 10^{-10} \mathrm{~m}$ as atomic length scale the wave functions are of the form ( $n=$ principal quantum number, $l=$ angular momentum quantum number, $m=$ magnetic quantum number)

$$
\Psi_{\text {mim }}(r, \vartheta, \varphi)=N_{n \cdot} \cdot e^{-r / n}\left(\frac{r}{n}\right) L_{m+i}^{21+1}(r) \cdot Y_{m}(\vartheta, \varphi)
$$

where $N_{N}$ is a normalization factor, $L_{n+1}^{2 / 1}(r)$ are the generalized Laguerre polynomials and $Y_{b m}$ the spherical harmonics. The wave functions are energetically degenerate with respect to $-l \leq m \leq+l$ and $l<n$. As an example, we consider the (field-free) wave function with principal quantum number $n=5$ and magnetic quantum number $m=0$. Furthermore, we have chosen a linear combination of the degenerate $l=0,2,4$ states in such a way that they possess the correct symmetry for a magnetic field (in the limit $B \rightarrow 0$ ). The wave function is axial symmetric with respect to the $z$-axis.

In Fig. Sa we first show lines of constant probability of presence in two orthogonal cuts ( $x z$ - and $x y$-plane). then the three-dimensional plots of the corresponding contour surfaces (Fig. 5b), and finally in Figs. 5c, d the results of our method employing a raster of 575 $\times 575$ lines-of-sight and 256 intensity levels. In the bottom pictures (Fig. 5d) the atom is rotated by $45^{\circ}$ around the $x$-axis (left) and additionally rotated by $45^{\circ}$ around the $z$-axis (right). The real extent of the hydrogen atom in this excited state is about $3 \mathrm{~nm}$.

To demonstrate the visualization power of the lineof-sight integration for a more complex physical system, in Fig. 6 we show the modulus of the wave function of highly excited (Rydberg) states of the hydrogen atom in a magnetic feld of $6 \mathrm{Tes}$. The wave functions were calculated by diagonalizing Hamiltonian matrices with dimension 6400 and with about $10^{6}$ nonvanishing elements. (For more details on the subject see [2].) These atoms with binding energies which are only some millielectronvolts below the ionization threshold are bizarre and highly sensitive structures with a spatial extent of up to $10^{-3} \mathrm{~mm}$.

\section{REFERENCES}

1. Special lssue on ViSC, Comp. Graphics 21(6), (1987).

2. G. Wunner and $\mathbf{H}$. Ruder, Atoms in strong magnetic fields. Physica Scripla 36, 291-299 (1987). 